$\begin{array}{lcr} & \text { Berechnet für } & \text { (iefunden } \\ & \mathrm{C}_{7} \mathrm{H}_{10} \mathrm{O}_{5} \mathrm{Ag}_{2} & \\ \mathrm{C} & 21,53 & 21,32 \\ \mathrm{H} & 2,59 & 2,63 \\ \mathrm{Ag} & 5 \tilde{5}, 31 & 5 \tilde{5}, 38\end{array}$

Aus diesen Salzen lässt sich die zweibasische Oxysäure nicht isoliren. Selbst als zur Zersetzung des Baryumsalzes weniger als die theoretische Menge Salzsäure angewandt, die Lösung mit Aether ausgeschüttelt und der Aether bei gewöhnlicher Temperatur verdunstet wurde, blieb doch nur die Lactonsäure zurück.

\title{
II. Einwirkung von Essigsäureanhydrid auf tricarballylsaures Natrium bei liegenwart vou Benzaldehyd;
}

von Wilhelm Sternberg.

Ketodilacton der Benzal- $\beta$-acetglutarsäure,

$$
\mathrm{C}_{14} \mathrm{H}_{12} \mathrm{O}_{4}=\mathrm{CH}_{3}-\mathrm{C} \sum_{0-\mathrm{CO}-\mathrm{C}=\mathrm{CH}-\mathrm{C}_{6} \mathrm{H}_{5}}^{\mathrm{CH}}
$$

In der Einleitung zu diesen Untersuchungen (Seite 3) ist bereits der Gedankengang erwähnt, von dem aus dieser Versuch anfänglich ausgeführt wurde.

Ein Gemisch von bei $140^{\circ}$ getrocknetem tricarballylsaurem Natrium, Essigsäureanhydrid und Benzaldebyd im Verhältniss ibrer Molekulargewichte wurde in einem mit Steig- und Chlorcalciumrohr versehenen Kolben circa 50 Stunden im Paraffnbade auf $120-130^{\circ}$ erhitzt. Später fanden wir, dass auch hier die Ausbeute erböht wird, wenn statt 1 Mol. Essigsäureanbydrid $1 \frac{1}{2}$ Mol. angewandt werden. Unter Entwickelung von Kohlensäure nahm das Gemisch bald eine gelbe, später dunkelbraune Färbung an. Nach dem Erkalten wurde die festgewordene Masse mit heissem Wasser behandelt. Es entstand eine trübe Lösung, aus der sich eine duukle, harzige Masse 
abschied. Ohne davon $z$ u trennen, wurde darauf durch Destillation im Dampfstrome der unveränderte Benzaldehyd entfernt, dann heiss filtrirt und die Lösung mit Chloroform ausgeschüttelt, bis dieses nichts mehr aufnahm. Aus der rückständigen Flüssigkeit konnte, auch nach dem starken Ansäuern, mit Chloroform nichts mehr extrahirt werden.

Die Chloroformausztige wurden nach dem Abdestilliren des Lösungsmittels mit Wasser versetzt und auf dem Wasserbade zur Vertreibung der Essigsäure wiederholt abgedampft. Der Rückstand, welcher im Vacuum nach einiger Zeit erstarrte, wurde mit Sodalösung in der Kälte behandelt, wobei sich ein Theil mit brauner Farbe löste, ein anderer, das gebildete Ketodilacton, aber als schmutzig weisser Körper ungelöst blieb. Nach dem Absaugen der Flüssigkeit und Auswaschen mit verdüntem Natriumcarbonat und Wasser blieb das Lacton fast farblos zurück. Durch mehrfaches Umkrystallisiren aus Alkohol oder Chloroform mit Aetherzusatz wurde es gereinigt und schmolz dann glatt bei $162^{\circ}$.

Die braune alkalische Lösung wurde mit Salzsäure versetzt und die sich abscheidende Schmiere der Flüssigkeit mit Aether entzogen. Es gelang jedoch nicht, ausser geringen Mengen Benzoësäure, einen gut charakterisirten Körper daraus zu erhalten, ebenso war ein solcher aus dem von der Flüssigkeit vorher getrennten Harzklumpen, der gepulvert lange Zeit mit verdünnter Essigsäure ausgekocht wurde, nicht mehr abzuscheiden.

Es musste nun auffallen, dass das inzwischen von Roth aufgefundene Ketodilacton $\operatorname{der} \boldsymbol{\beta}$-Acetglutarsäure sich bei dieser Condensation nicht gebildet haben sollte und wicht mit in das Chloroform übergegangen war. Da dieses Lacton jedoch durch Wasseraufnahme leicht in die Ketonsäure übergeht und letatere nicht leicht löslich in Chloroform ist, so kounte dennoch diese Verbindung vorhanden sein.

Um darauf zu prüfen, wurde die mit Chloroform extrahirte Flüssigkeit mit starker Salzsäure eingedampft, der fast weisse 
Rückstand getrocknet, gepulvert und mit Chloroform behandelt. Die eingeengten Auszüge schieden nach einigem Stehen Krystallnadeln $a b$, die die ganze Lösung durchsetzten, nach dem Umkrystallisiren den Schmelzp. $99^{\circ}$ und alle anderen Eigenschaften des in der vorstehenden Abhandlung beschriebenen Ketodilactons der Acetglutarsäure besassen. Die Quantität desselben betrug etwa das vier- bis füffache von der des gleichzeitig entstandenen Ketodilactons der Benzalacetglutarsäure, welches bei allen Versuchen nur in verhältnissmässig geringer Menge (bei Anwendung von $25 \mathrm{~g}$ tricarballylsauren Natriums höchstens $1 \mathrm{~g})$ gebildet wurde.

In dem Salzrückstande, aus welchem mit Chloroform das Ketodilacton der Acetglutarsäure ausgezogen war, konnte ausser Essigsäure als einzige organische Verbindung nur noch unveränderte Tricarballyisäure nachgewiesen werden, welche daraus durch Extrabiren mit Aether zurückgewonnen wurde.

Das Ketodilacton der Benzal- $\beta$-acetglutarsäure stellt gut ausgebildete, glänzende Krystallblättchen dar, die constant bei $162^{0}$ schmelzen urd sich auch weit über dem Schmelzpunkt nicht zersetzen. Es wird von kaltem Wasser und selbst von kochendem nicht aufgenommen. Chloroform, Alkohol, Benzol, Aceton und Essigsäure, zumal in der Wärme angewandt, sind gute Lösungsmittel. In Aether, Schwefelkohlenstoff und Ligroin ist das Iuacton hingegen nicht löslich. Natriumcarbonatlösung greift die Verbindung selbst bei sehr langem Kochen nicht merklich an.

Zur Analyse wurde das aus Alkohol umkrystallisirte, über Schwefelsäure bis zur Gewichtsconstanz getrocknete Lacton verwandt.

I. $0,2007 \mathrm{~g}$ gabes $0,5035 \mathrm{CO}_{2}$ and $0,094 \mathrm{H}_{2} \mathrm{O}$.

II. $0,2262 \mathrm{~g}, \quad 0,5712 \mathrm{CO}_{2} \quad, \quad 0,1055 \mathrm{H}_{2} \mathrm{O}$.

\begin{tabular}{ccrc} 
& Berechnet fïr. & \multicolumn{2}{c}{ Gefunden } \\
\cline { 3 - 4 } & $\mathrm{C}_{14} \mathrm{H}_{12} \mathrm{O}_{4}$ & I. & II. \\
$\mathrm{C}$ & 68,85 & 68,42 & 68,87 \\
$\mathrm{H}$ & 4,92 & 5,24 & 5,18
\end{tabular}


Benzal - $\beta$ - acetglutarsäure.

Beim Kochen mit starken Basen liefert das Ketodilacton die Salze der Benzal- $\beta$-acetglutarsäure. Es ist uns indess nicht gelungen, aus diesen die freie Säure in reinem Zustande zu isoliren, weil deren Rückverwandlung in das Lacton schon bei gewöhnlicher Temperatur erfolgt. Als wir die Lösung des Calciumsalzes mit weniger als der erforderlichen Menge verdünnter Salzsäure versetzten, liess sich mit Aether die Säure ausschütteln, aber die ätherischen Auszüge hinterliessen beim freiwilligen Verdunsten eine zäbe, durchsichtige Masse, welche im Exsiccator alimählich krystailinisch erstarrte. Beim Versuch, diese von Neuem in absolutem Aether zu lösen, blieb sehr viel des Ketodilactons ungelöst und die davon getrennte ätherische Lösung verbielt sich beim Verdunsten wieder genau wie das erste Mal. Beim jedesmaligen Aufösen des Rückstandes in Aether blieb zuruckgebildetes Ketodilacton unge!öst, so dass auf diese Weise schliesslich die ganze Menge der Säure in das I acton zurückgeführt werden kann.

Salze der Benzal- $\beta$-acetglutarsäure.

C'alciumsalz, $\mathrm{C}_{14} \mathrm{H}_{12} \mathrm{O}_{5} \mathrm{Ca}+1 \frac{1}{2} \mathrm{H}_{2} \mathrm{O}$. Das Ketodilacton wurde mit dünner Kalkmilch eive Stunde gekocht, dann filtrirt, der überschüssige Kalk durch Kohlensäure entfernt und die Lösung verdunstet. Beim Stehen der concentrirten Lösung im Exsiccator trocknet sie zu einem allmählich erhärtenden Syrup ein, aber wenn man die concentrirte Lösung zum Sieden erwärmt, scheidet sich das Salz zum grössten Theil pulverig aus. Dieses Saiz wurde analysirt.

$0,305 \mathrm{~g}$ des luftrocknen salzes verloren bei $120^{\circ} 0,027 \mathrm{H}_{2} \mathrm{O}$ und gaben $0,1274 \mathrm{SO}_{4} \mathrm{Ca}$.

Berechnet für

$$
\mathrm{C}_{3} \mathrm{H}_{28} \mathrm{O}_{5} \mathrm{Ca}
$$

$\mathrm{Ca} \quad 13,33$

$11 / 2 \mathrm{H}_{2} \mathrm{O} \quad 8,56$

Baryumsalz, $\mathrm{C}_{14} \mathrm{H}_{12} \mathrm{O}_{6} \mathrm{Ba}+\mathrm{H}_{2} \mathrm{O}$. Die Darstellung war die nämliche wie beim Calciumsalz. Das Salz konnte aus Wasser 
nicht krystallisirt erhalten werden. Der wässrigen Lösung wurde soviel Alkohol zugegeben, bis der entstandene Niederschlag in der IIitze eben gelöst wurde. Beim Erkalten der Lösung schied sich das Salz amorph ab. Es wurde abgesaugt, ausgewaschen und lufttrocken aualysirt.

I. $0,2507 \mathrm{~g}$ verloren bei $120^{\circ} 0,0095 \mathrm{H} \mathrm{O}$ und gaben $0,142 \mathrm{SO}_{4} \mathrm{Ba}$.

II. 0,1915 g nahmen bei $120^{\circ} 0,0085$ an Gewicht ab.

Berechnet für

$$
\mathrm{C}_{14} \mathrm{H}_{12} \mathrm{O}_{6} \mathrm{Ba}
$$

Ba

1 Iol. $\mathrm{H}_{2} \mathrm{O} \quad 4,34$

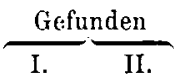

$34,61-$

$3,79 \quad 4,43$

Silbersalz, $\mathrm{C}_{14} \mathrm{H}_{12} \mathrm{O}_{5} \mathrm{Ag}_{2}$. Das Salz fiel bei Zugabe von Silbernitrat zur Lösung des Calciumsalzes als käsiger Niederschlag aus. Dieser musste sofort filtrirt werden, da andernfalls Zersetzung eintrat. Es ist ziemlich wasserlöslich, bei Anwendung von Wärme tritt unter Braunfärbung Zersetzung ein, das Filtrat enthält dann nur Spuren des Salzes. Es wurde daher, um analysenreines Salz zu erhalten und um Verluste möglichst $z u$ vermeiden, die Calciumsalzlösung mit der berechneten Menge Silbernitrat versetzt.

Der Niederschlag wurde abgesaugt, mit sehr kleinen Quantitäten Wasser öfters ausgewaschen, über Schwefelsäure im dunklen Luftpumpenexsiccator getrocknet und analysirt.

I. 0,183 g gaben $0,2348 \mathrm{CO}_{2}, 0,049 \mathrm{H}_{2} \mathrm{O}$ und $0,0816 \mathrm{Ag}$.

II. $0,2084 \mathrm{~g} \quad, \quad 0,0948 \mathrm{Ag}$.

\begin{tabular}{lcrc} 
& Berechnet für & \multicolumn{3}{c}{ (iefunden } \\
\cline { 2 - 4 } & $\mathrm{C}_{14} \mathrm{H}_{12} \mathrm{O}_{5} \mathrm{Ag} \mathrm{g}_{2}$ & \multicolumn{1}{c}{$\frac{\mathrm{I}}{\mathrm{Il}}$} \\
$\mathrm{H}$ & 35,29 & 35,00 & - \\
$\mathrm{H}$ & 2,52 & 2,94 & \\
$\mathrm{Ag}$ & 45,38 & 44,59 & 45,48
\end{tabular}

Dibromid des Ketodilactons, $\mathrm{C}_{14} \mathrm{I}_{12} \mathrm{Br}_{2} \mathrm{O}_{4}$. Um die Richtigkeit der oben für das Ketodilacton aufgestellten Formel zu prüfen, Laben wir seine Additionsfähigkeit gegen Brom untersucht. Wenn man zu der mit Eiswasser abgekühlten Lösung des Ketodilactons in Chloroform unter Umschütteln eine Lösung von Brom (ein Mol.) in dem zehufachen Gewicht Chloroform 
tropfenweise zufliessen lässt, so ist zunächst eine Reaction nicht wahrzunehmen, und erst, als nach Zusatz der ganzen Brommenge die Flüssigkeit sich selbst überlassen wurde, trat im Verlauf von zwei bis drei Tagen fast völlige Entfärbung ein. Das Chloroform wurde dann im Vacuum entfernt und der Rückstand über Aetznatron von etwas noch vorhandenem Brom und Bromwasserstoff befreit. Die Masse besass dann eine fast weisse Farbe und krystallinische Beschaffenheit. Sie wurde in reinem Benzol in der Wärme gelöst; beim Erkalten schieden sich nadelförmige Kryställchen aus, die unter Zersetzung bei $163^{\circ}$ schmolzen.

Die Verbindung ist leicht löslich in Chloroform; von Aether, Schwefelkohlenstoff und Ligroïn wird sie nicbt aufgenommen.

$0,283 \mathrm{~g}$, in Vacuum getrocknet, gaben 0,2641 AgBr.

$\begin{array}{ccc} & \text { Berechnet für } & \text { Gefunden } \\ & \mathrm{C}_{14} \mathrm{H}_{12} \mathrm{Br}_{2} \mathrm{O}_{4} & \\ \mathrm{Br} & 39,60 & 39,70\end{array}$

Die Verbindung war demnach das erwartete Dibromid.

Reduction des Ketodilactons. Obgleich das Ketodilacton verhältnissmässig leicht Brom addirt, setzt es auffälliger Weise der Anlagerung von Wasserstoff einen ausserordentlich grossen Widerstand entgegen und bei Anwendung von Natriumamalgam ist es uns überhaupt nicht gelungen, ein Reductionsproduct $z u$ isoliren. Wir haben bei wiederholten Versuchen eine immer grössere Menge von Natriumamalgam - zuletzt auf 1,8 $\mathrm{g}$ des Lactons $2,5 \mathrm{~kg}$ vierprocentigen Amalgams - angewandt und während der Reaction einen langsamen Strom von Kohlensäure durch die Flüssigkeit geleitet, aber das nachher mit Aether extrahirte Product zeigte immer das Verhalten des Productes, welches wir bei den vorhin beschriebenen Versuchen zur Gewinnung der Benzalacetglutarsäure beobachtet hatten und durch wiederholtes Behandeln mit Aether und Verdunsten der Lösung im Vacuum liess sich daraus regelmässig sehr annähernd die ganze Menge des angewandten Ketodilactons unverändert wieder gewinnen.

Annalen der Chomio 314. Bd. 
$\mathrm{Zu}$ einem besseren Resultate führte die Anwendung von Zinkstaub in essigsaurer Lösung, aber als wir bei einem ersten Versuche $2 \mathrm{~g}$ des Lactons in $80 \mathrm{ccm}$ Essigsäure und $50 \mathrm{ccm}$ Wasser gelöst, mit $5 \mathrm{~g}$ Zinkstaub versetzt und sechs Stunden lang am Rückflusskühler gekocht hatten, schied sich beim Stehen die grösste Menge des angewandten Lactons in langen, Farrenwedeln gleichenden Krystallen unverändert wieder $a b$. Es wurden darauf $4 \mathrm{~g}$ des Ketodilactons in $160 \mathrm{ccm}$ Eisessig und $100 \mathrm{ccm}$ Wasser gelöst und nach und nach $75 \mathrm{~g}$ Zinkstaub zugesetzt. Die Flüssigkeit wurde eine volle Woche hindurch am Rückflusskühler im lebhaften Sieden erhalten, bis das Zink anähernd aufgebraucht war.

In dem Reactionsproducte machte sich eine Krystallabscheidung nicht bemerklich, sodass hieraus gefolgert werden konnte, dass ein in Essigsäure leichter löslicher Körper entstauden war. Die Flüssigkeit wurde dann mit Chloroform extrahirt und der vom letzteren befreite Auszug behufs Verjagung der Essigsäure mit Wasser öfters abgedampit. Das chocoladenfarbige, im Vacuum festgewordene Product hatte ein Gewicht von annähernd $4 \mathrm{~g}$. Es zeigte sich auch, dass nach dem Ansäuern der Flüssigkeit und Ausziehen mit Cbloroform nichts mehr in letzteres überging, ebenso liess sich aus dem vorher abfiltrirten Zink, welches in Salzsäure gelöst wurde, nichts mehr extrahiren.

Der Rückstand wurde nun fein gepulvert und, um ihn reiner zu bekommen, mit kleinen Mengen absoluteu Aethers ausgewaschen. Er war dann sehr leicht löslich in Chloroform und Alkohol, weniger leicht in Aether. Es konnten jedoch keine charakteristischen Krystalle erhalten werden und es zeigte sich bald, dass das Product kein einheitliches war. Benzol war das beste Lösungsmittel, welches eine Trenuung dieses Gemisches von zwei Körpern ermöglichte. Eine nicht zu concentrirto Lösung darin scheidet nach einigem Stehen in der Kälte compacte, zu Drusen vereinigte Krystalle aus, von denen man im geeigneten Momente die überstehende Flüssigkeit abgiessen 
muss. Im anderen Falle werden die Krystalle von kleinen, stecknadelkopfgrossen, weissen Kugeln durchsetzt, sodass eine mechanische Trennung mit Iülfe der Lupe grosse Schwierigkeiten bereitet. Ein noch längeres Stehenlassen der Flüssigkeit hat zur Folge, dass dieser zweite Körper sich sandig niederschlägt; man kann ihn dann, durch Umschwenken der Lösung in dieser suspendirt, von dem anderen abgiessen.

Es gelingt so, durch fractionirtes Krystallisiren und durch Auslesen der verschiedenen Krystalle mechanisch die Körper annähernd zu trennen. Der zuerst aus dem Benzol abgeschiedene Körper wurde durch wiederholtes Umkrystallisiren völlig rein erhalten und schmolz dann glatt bei $134^{\circ}$. Die zweite Verbindung wurde aus Alkohol umkrystallisirt. Die ersten Ausscheidungen sind stets rein, während die letzten den anderen Körper beigemengt enthalten. Dieses zweite Product schmilzt ohne Zersetzung constant bei $169^{\circ}$. Es hat den Anschein, als ob beide Verbindungen $z u$ gleichen Gewichtstheilen im Gemisch vorhanden seien.

Durch die Analyse erwies sich, dass die beiden Verbindungen isomerisch sind und die Zusammensetzung $\mathrm{C}_{\mathbf{1 4}} \mathrm{H}_{14} \mathrm{O}_{4}$ haben. Beide sind neutrale Körper. Wir bezeichnen sie als $\alpha$ - und $\beta$-Ketodilactone der Benzyl- $\beta$-acetglutarsäure.

$\alpha$-Ketodilacton der Benzyl- $\beta$-acetglutarsäure.

Das Lacton ist leicht löslich in heissem Benzol, Chloroform, Aethyl-, Methyl-Alkohol und Aceton, weniger leicht in Aether und in den genannten Lösungsmitteln ohne Anwendung von Wärme. Von Schwefelkohlenstoff und Ligroin wird es ebenso wie von kaltem Wasser und Natriumcarbonat nicht gelöst. In heissem Wasser ist es etwas löslich mit saurer Reaction; leicht wird es hingegen von verdünnter Essigsäure gelöst. Es schmilzt constant bei $134^{\circ}$.

Das aus Benzol krystallisirte Lacton wurde analysirt.

I. $0,219 \mathrm{~g}$ gaben $0,3481 \mathrm{CO}_{2}$ und $0,1131 \mathrm{H}_{2} \mathrm{O}$.

II. $0,2453 \mathrm{~g} \quad, \quad 0,613\left(\mathrm{C}_{2}\right.$ and $0,1307 \mathrm{H}_{2} \mathrm{O}$. 


\begin{tabular}{ccrr} 
& Berechnet für & \multicolumn{2}{c}{ Gefunden } \\
\cline { 3 - 3 } & $\mathrm{C}_{14} \mathrm{H}_{14} \mathrm{O}_{4}$ & I. & II. \\
C & 68,29 & 68,25 & 68,15 \\
H & 5,69 & 5,73 & 5,92
\end{tabular}

Beim Kochen mit starken Basen liefert das Lacton die Salze der Benzyl- $\beta$-acetglutarsäure, aber diese selbst zu isoliren ist uns nicht gelungen, weil sie eben so leicht sich in das Lacton zurückverwandelt, wie die Benzal- $\beta$-acetglutarsäure.

Calciumsalz, $\mathrm{C}_{1.4} \mathrm{H}_{14} \mathrm{O}_{5} \mathrm{Ca}+2 \mathrm{H}_{2} \mathrm{O}$. Das Ketodilacton wurde cine Stunde lang mit Kalkmilch gekocht, der überschüssige Kalk durch Einleiten von Kohlensäure ausgefällt und die filtrirte Lösung auf dem Wasserbade bis zur beginnenden Abscheidung des Salzes an der Oberfäche eingedampft. Beim Stehenlassen in der Kälte krystallisirte es nicht aus, jedoch entstand nach längerem Stehen im Vacuum ein krystallinischer Niederschlag.

Zur Analyse diente das 24 Stunden lang an der Luft getrocknete Salz.

0,2058 g verloren bei $140^{\circ} 0,022 \mathrm{H}_{2} \mathrm{O}$ und gaben $0,0818 \mathrm{SO}_{4} \mathrm{Ca}$.

Berechnet für $\mathrm{C}_{14} \mathrm{H}_{14} \mathrm{O}_{5} \mathrm{Ca}$

Ca 13,24

$2 \mathrm{H}_{2} \mathrm{O} \quad 10,65$
Gefunden

13,08

10,69

Baryumsalz, $\mathrm{C}_{14} \mathrm{H}_{14} \mathrm{O}_{5} \mathrm{Ba}+2 \mathrm{H}_{2} \mathrm{O}$, wurde wie das Calciumsalz bereitet. Es ist sehr leicht löslich. Im Vacuum trocknete die Lösung zu einem Syrup eín, der später crstarrte.

Es wurde in einem Gemisch von $2 / 3$ Volumen Alkohol und $1 / 3$ Volumen Wasser in der Wärme gelöst; beim Erkalten der Lösung entstand dann eine sandige Abscheidung.

$0,4244 \mathrm{~g}$ des lufttrocknen Salzes verloreu bei $140^{\circ} \quad 0,051 \mathrm{H}_{2} \mathrm{O}$ und gaben $0,223 \mathrm{sO}_{4} \mathrm{Ba}$.

$\begin{array}{lcc} & \text { Berechnet für } & \text { Gefunden } \\ & \mathrm{C}_{14} \mathrm{H}_{14} \mathrm{O}_{5} \mathrm{Ba} & \\ \mathrm{Ba} & 34,33 & 34,64 \\ 2 \mathrm{H}_{2} \mathrm{O} & 11,92 & 12,02\end{array}$

Das Silbersalz, $\mathrm{C}_{14} \mathrm{H}_{14} \mathrm{O}_{5} \mathrm{Ag}_{3}$, wurde aus dem Calciumsalz mittelst salpetersauren Silbers hergestellt. Es fällt als dicker, 
weisser Niederschlag aus, der öfters mit kleinen Mengen Wassers ausgewaschen und im Vacuum getrocknet wurde. Von kaltem Wasser wird das Salz nicht aufgenommen und auch in heissem löst es sich nur sehr wenig. Trocken ist es lichtbeständig.

$0,5018 \mathrm{~g}$ des bei $80^{\circ}$ getrockneten Salzes gaben $0,6 \overline{\mathrm{CO}_{2}}, 0,1347$ $\mathrm{H}_{2} \mathrm{O}$ und $0,2348 \mathrm{Ag}$.

Berechnet für

$$
\mathrm{C}_{14} \mathrm{H}_{14} \mathrm{O}_{5} \mathrm{Ag}
$$

C $\quad 35,22$

$\mathrm{H} \quad 2,93$

Ag $\quad 45,07$ (iefinden

35,32

2,97

45,32

$\beta$-Ketodilacton der Benzyl- $\beta$-acetglutarsüure.

In reinem Zustande ist es etwas schwerer löslich in heissem Alkohol, Aceton, Chloroform und Benzol als das rorher beschriebene. Aus letzterem Lösungsmittel fällt es beim Erkalten krystallinisch aus. In Chloroform gelöst, scheiden sich an der Oberfläche kleine quadratische Plätchen aus, die zu einer festen Krystalldecke zusammenschiessen. Aus Alkohol krystallisirt dic Verbindung in Form von sternartig gruppirten Blättchen, die denen des ungesättigten Lactons sehr äbnlich sehen. Mit dem Isomeren hat es ferner die Eigenschaft gemein, dass es löslicher in verdünnter Essigsäure ist als das ungesättigte Lacton, ebenso ist es wie jenes etwas löslich in Aether. Von Schwefelkohlenstoff, Ligroïn und Natriumcarbonatlösung wird es nicht aufgenommen. Es schmilzt bei $169^{\circ}$.

I. $0,2412 \mathrm{~g}$ gaben $0,6006 \mathrm{CO}_{2}$ and $0,1278 \mathrm{H}_{2} \mathrm{O}$.

II. $0,2507 \mathrm{~g} \quad, \quad 0,6295 \mathrm{CO}_{2} \quad, \quad 0,1281 \mathrm{H}_{2} \mathrm{O}$.

\begin{tabular}{lcrr} 
& Berechnet für & \multicolumn{2}{c}{ Utefunden } \\
& $\mathrm{C}_{14} \mathrm{H}_{14} \mathrm{O}_{4}$ & I. & II. \\
$\mathrm{C}$ & 68,29 & $67,9 \pm$ & 68,48 \\
$\mathrm{H}$ & 5,69 & 5,88 & 5,67
\end{tabular}

Gegen Basen verhält es sich wie die isomere Verbindung und aus den Salzen der Ketonsäure wird es unverändert wieder gewonnen. Die Säure selbst haben wir ebenfalls nicht in reinem Zustande isoliren können. 
Calciumsalz, $\mathrm{C}_{14} \mathrm{H}_{14} \mathrm{O}_{5} \mathrm{Ca}+2 \frac{1}{2} \mathrm{H}_{2} \mathrm{O}$. Aus der concentrirten Lösung scheidet sich das Salz in der Hitze krystallinisch $a b$. Es ist dann in grossen Wassermengen äusserst schwer wieder auflösbar.

$0,3084 \mathrm{~g}$ des durch 24 Strunden langes Liegen an der Luft getrockneten Salzes verloren bei $140^{\circ} 0,04013 \mathrm{H}_{2} \mathrm{O}$ und gaben 0,1183 $\mathrm{SO}_{4} \mathrm{Ca}$.

Berechnet für

$$
\mathrm{C}_{14} \mathrm{H}_{14} \mathrm{O}_{5} \mathrm{Ca}
$$

$\mathrm{Ca} \quad 13,24$

$2^{1},{ }_{2} \mathrm{H}_{2} \mathrm{O} \quad 12,96$

\section{Gefunden}

12,97

13,26

Baryumsalz, $\mathrm{C}_{14} \mathrm{H}_{14} \mathrm{O}_{6} \mathrm{Ba}+4 \mathrm{H}_{2} \mathrm{O}$. Die Lösung des Salzes trocknet im Vacuum zu einem später erstarrenden Syrup ein. Es wurde in der Hitze in einem Gemisch von gleichen Theilen Alkohol und Wasser gelöst, aus welchem es nach längerer Zeit in der Kälte als weisses, krystallinisches Pulver ausfiel. Zur Analyse diente das 24 Stunden lang der Luft ausgesetzte Salz.

$0,5594 \mathrm{~g}$ verloren bei $140^{\circ} 0,0888 \mathrm{H}_{2} \mathrm{O}$ und gaben $0,273 \mathrm{SO}_{4} \mathrm{Ba}$.

Berechnet für

$$
\mathrm{C}_{14} \mathrm{H}_{14} \mathrm{O}_{5} \mathrm{Ba}
$$

$\mathrm{Ba} \quad 34,33$

$4 \mathrm{H}_{2} \mathrm{O} \quad 15,29$
Gefunden

34,12

15,87

Silbersalz, $\mathrm{C}_{14} \mathrm{H}_{14} \mathrm{O}_{5} \mathrm{Ag}_{2}$. Der aus dem Calciumsalz mit Silbernitrat entstandene Niederschlag wurde im Vacuum, vor Licht geschützt, getrocknet. Vom Wasser wird das Salz beim Kochen kaum aufgenommen; die eingeengte Lösung scheidet bei längerem Stehen im Exsiccator braune Flocken ab.

$0,3986 \mathrm{~g}$ des bei $80^{\circ}$ getrockneten Salzes gaben $0,5114 \mathrm{CO}_{2}, 0,1023$ $\mathrm{H}_{2} \mathrm{O}$ und $0,1802 \mathrm{Ag}$.

$\begin{array}{cr}\text { Berechnet für } & \text { Gefunden } \\ \mathrm{C}_{14} \mathrm{H}_{14} \mathrm{O}_{5} \mathrm{Ag}: & \\ 35,22 & 34,99 \\ 2,93 & 2,85 \\ 45,07 & 45,21\end{array}$

Die Versuche, diese gesättigten Ketodilactone durch weitere Reduction in die entsprechenden Lactonsäuren überzuführen, 
konnten wegen Mangels an Material nicht zu einem sichereu Endresultate durchgeführt werden.

\title{
III. Iarstellung des Ketodilactons der Henzal- $\beta$-aceiglutar- säure aus dem hetodilacton der $\beta$-Acetglutarsäure;
}

\author{
von Ernst Roth.
}

Die in der vorstehenden Abhandlung beschriebenen Versuche machen es sehr wahrscheinlicb, dass die Einwirkung des Benzaldehyds eine secundäre Reaction ist, dass zunächst nur das Essigsäureanbydrid mit dem tricarballylsauren Natrium reagirt und das so gebildete Ketodilacton sich zum Theil nachher mit dem Benzaldehyd unter Wasseraustritt condensirt. Um dies zu prüfen, wurde das Ketodilacton der $\beta$-Acetglutarsäure $(5,2 \mathrm{~g})$ mit Benzaldehyd $(3,6 \mathrm{~g})$ und Essigsäureanhydrid $(3,4 \mathrm{~g})$ zu gleichen Molekülen im Paraffinbade drei Tage auf $120^{\circ}$ erhitzt. Das Ketodilacton löste sich in dem Gemisch auf und nachher krystallisirten aus der schwach gefärbten Flüssigkeit beim Erkalten lange Nadeln aus, die sich in heissem Wasser leicht lösten. Der freie Aldelyd wurde mit Wasserdämpfen abdestillirt und die wässrige Lüsung mit Chloroform ausgezogen. Ich erhielt nur unverändertes Lacton und kleine Mengen von Benzoësäuren, aber nicht das von Sternberg studirte Lacton.

Da dieser Versuch das gewünschte Resultat nicht ergab, wurde ein zweiter unter sonst gleichen Bedingungen mit dem trocknen Natriumsalz der $\beta$-Acetglutarsäure $(7,30 \mathrm{~g})$ ausgeführt. Die Reactionsmasse wurde fest und nur wenig braun gefärbt. Nachdem der überschüssige Aldehyd abdestillirt war, wurde die wässrige Lösung mit Salzsäure angesäuert, das vorhandene Harz mechanisch von der Flüssigkeit getrennt und letztere mit Chloroform ausgezogen. Der vom Chloroform befreite Rückstand wurde aus Alkohol umkrystallisirt und lieferte schöne glänzende Blättchen, welche bei $162^{\circ}$ schmolzen. Die Ausbeute betrug $0,9 \mathrm{~g}$, d. i. $12,24 \mathrm{pC}$. der theoretischen. 